\title{
Size and shape fluctuations of ultrasoft colloids
}

\author{
Huarui Wu $\odot,{ }^{1}$ Kun Song, ${ }^{1}$ Wei-Ren Chen $\odot,{ }^{2}$ Jing Song, ${ }^{1}$ Lionel Porcar, ${ }^{3}$ and Zhe Wang $\oplus^{1, *}$ \\ ${ }^{1}$ Department of Engineering Physics and Key Laboratory of Particle and Radiation Imaging (Tsinghua University) \\ of Ministry of Education, Tsinghua University, Beijing 100084, China \\ ${ }^{2}$ Neutron Scattering Division, Oak Ridge National Laboratory, Oak Ridge, Tennessee 37831, USA \\ ${ }^{3}$ Institut Laue-Langevin, BP 156, F38042 Grenoble CEDEX 9, France
}

(Received 19 January 2021; accepted 3 September 2021; published 22 September 2021)

\begin{abstract}
An ultrasoft colloidal particle fluctuates due to its flexibility. Such fluctuation is essential for colloidal structure and dynamics, but is challenging to quantify experimentally. We use dendrimers as a model system to study the fluctuation of ultrasoft colloids. By considering the dynamic polydispersity in the small-angle neutron scattering (SANS) model and introducing the fluctuation of invasive water into the contrast in SANS, we reveal the fluctuating amplitudes of the size and shape of the dendrimer of generation 6 at finite concentrations. The size fluctuation is suppressed while the shape fluctuation increases as the weight fraction of dendrimers passes $11 \%$. With neutron spin echo data, we suggest that such a crossover originates from the competition between the interand intraparticle dynamics. Further investigation on lower-generation samples shows a contrary result, which suggests a structural basis for these dynamic phenomena.
\end{abstract}

DOI: 10.1103/PhysRevResearch.3.033271

\section{INTRODUCTION}

Ultrasoft colloids, such as dendrimers and star polymers, are featured by extraordinary molecular flexibility [1-6]. The amount of elastic energy stored by such a particle that undergoes a large strain can be just hundreds of or even tens of the thermal energy [7-10], which distinguishes it from emulsions, most microgels, or other common deformable particles [10-17]. Consequently, ultrasoft colloids exhibit significant thermal-activated molecular fluctuations. These fluctuations are crucial in many physical processes. Simulations suggest that the size and shape fluctuations considerably affect the self-diffusion of particles [18]. At concentrations close to the random close packing, it is proven that the shape fluctuation is related to the stress releasing and building of the particle and plays an essential role in the unusual dynamics [19]. Taking star polymers as the model ultrasoft system, many studies demonstrate that the molecular fluctuations facilitate the long-time relaxation of the local structure and contribute to the unique rheological phenomena of star polymer glasses $[10,20,21]$. Moreover, theoretical analysis [22] suggests that the arm-end fluctuations can enhance the particle displacement and consequently suppress the crystalline ordering in star solutions [23]. On the practical level, fluctuations can modulate the particle conformation [24] that profoundly impacts a large variety of applications [25-28]. For instance,

\footnotetext{
*Corresponding author: zwang2017@mail.tsinghua.edu.cn

Published by the American Physical Society under the terms of the Creative Commons Attribution 4.0 International license. Further distribution of this work must maintain attribution to the author(s) and the published article's title, journal citation, and DOI.
}

dendrimers have been established as drug carriers. The conformational fluctuation directly affects the size, shape, and internal cavity of the molecule, which are important for the drug loading and release ability and the permeability across the biomembrane [29-33]. Therefore, there is a strong need to quantify the size and shape fluctuations to advance our understanding of both the physics and applications of ultrasoft colloids.

In the past two decades, the neutron spin echo (NSE) technique has been used to measure the intraparticle motion of ultrasoft colloids at the dilute limit, and the fluctuations of the structural unit and shape of the isolated particle were revealed $[34,35]$. However, many ultrasoft colloidal suspensions of physical and technological importance are with finite concentrations $[10,19,36]$. For these systems, the NSE analysis of the molecular fluctuation is greatly complicated by the difficulty in experimentally determining the collective translational diffusion of particles and its coupling to the intraparticle dynamics [37]. On the other hand, the fluctuations influence the distribution of the particle conformation, which could be reflected in small-angle scattering (SAS) patterns. Note that most previous SAS studies of ultrasoft colloids have not explicitly considered the effect of particle size and shape fluctuations $[1,38]$. Many SAS analyses deteriorate at volume fractions higher than about $10 \%$. For example, the calculated SAS curve may not match the position or height of the main peak [7,9] or underestimate the intensity at small $Q(Q$ is the scattering vector) $[39,40]$. Thus, it is possible that the fluctuation effect is important in interpreting the SAS data of ultrasoft colloids and can be extracted by reasonable modeling.

In this work we study the size and shape fluctuations of ultrasoft colloids using neutral poly(amidoamine) (PAMAM) dendrimers dissolved in water as the model system. By introducing the dynamic polydispersity effect into the small-angle 

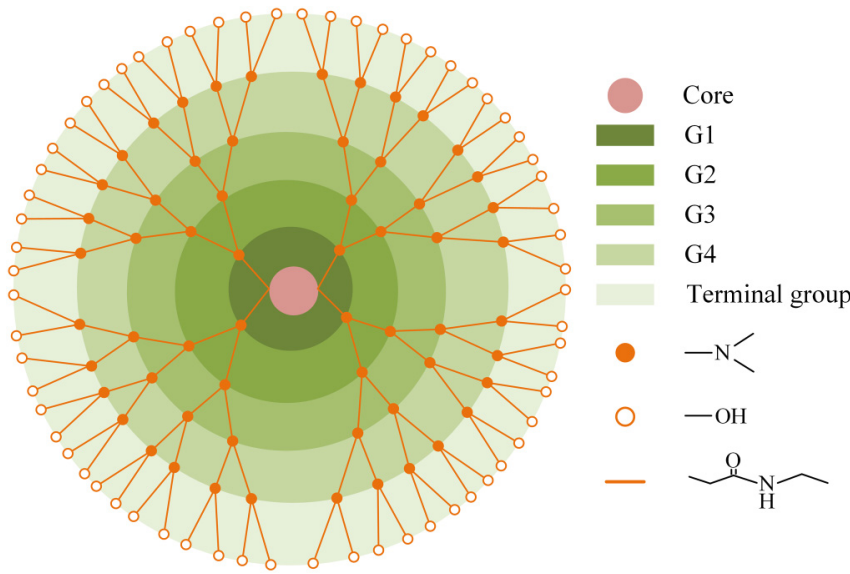

FIG. 1. Illustration of the molecular structure of the G4-OH PAMAM dendrimer [42].

neutron scattering (SANS) model, we are able to quantify the amplitudes of these fluctuations. It is seen that explicitly considering the size fluctuation is of special importance in interpreting the SANS patterns. The existence of the size fluctuation is further verified by incorporating the fluctuation of invasive water into the contrast-variation SANS analysis. Moreover, with the NSE results and the investigation into the lower-generation samples, we reveal the dependences of these fluctuations on the molecular structure and dynamics.

\section{EXPERIMENT}

The biomedical grade generation 4 and 6 (G4 and G6) PAMAM dendrimers with hydroxyl terminal groups and ethylenediamine cores were purchased from Dendritech, Inc., Midland, MI, USA. Dendrimers are regularly branched, treelike macromolecules with branches radially emanating from a central core $[41,42]$. The molecular structure of the G4 dendrimer is illustrated in Fig. 1. As the generation grows, the number of branching units grows exponentially. The G6 dendrimer, which is not illustrated here, has four times as many branches as the $\mathrm{G} 4$ dendrimer. The radii of gyration $\left(R_{\mathrm{g}}\right)$ of the G4 and G6 dendrimers in dilute solutions are around 2.0 and $2.4 \mathrm{~nm}$, respectively. These sizes are much smaller than those of the commonly used soft particles, e.g., microgels, emulsions, and grafted core-shell colloids. Thus, the fluctuation effect is expected to be more considerable in dendrimers. Their ultrasoftness can be characterized by a Gaussian-like interparticle pair potential with an amplitude tens times the thermal energy [9]. Deuterium oxide $\left(\mathrm{D}_{2} \mathrm{O}\right)$ was acquired from Cambridge Isotope Laboratories, Inc., Andover, MA, USA. The samples were prepared by dissolving PAMAM dendrimers in aqueous solutions according to the predetermined dendrimer weight fraction $c(c=1,5,10,12.5$, 15 , and $20 \mathrm{wt} . \%)$. Solvents used for the contrast-variation SANS experiments were prepared by mixing $\mathrm{D}_{2} \mathrm{O}$ and deionized water (purified from Millipore system) with the molar fraction of $\mathrm{D}_{2} \mathrm{O}$ in solvent $(\gamma)$ at $100 \%, 90 \%, 80 \%, 70 \%$, and $60 \%$.

Small-angle neutron scattering measurements were performed at the EQ-SANS instrument at the Spallation Neutron
Source at the Oak Ridge National Laboratory and at the D22 SANS instrument at the Institut Laue-Langevin in Grenoble, France. All the samples were contained in Hellma quartz cells of $1 \mathrm{~mm}$ path length at $20.0^{\circ} \pm 0.1^{\circ}$. Following standard data reduction procedures [43], the measured scattering intensity $I(Q)$ was corrected for detector background, detector sensitivity, sample transmission, and empty cell scattering.

\section{RESULTS AND DISCUSSION}

Dendrimers are nearly monodisperse macromolecules synthesized via well-controlled chemical reactions [44]. Traditionally, the SAS spectrum of monodisperse dendrimers is analyzed by decomposing the intensity into a product of a form factor describing the structure of a single particle and a structure factor characterizing the interparticle spatial correlation $[1,38]$. Nevertheless, dendrimers with the same molecular weight possess different conformations due to molecular fluctuations. Thus, dendrimer solutions should be regarded as polydisperse and the aforementioned simple decomposition may need to be improved. To reflect this dynamic polydispersity effect [45], we employ the well-known $\beta$ approximation for the SANS intensity of polydispersity [46]

$$
I(Q)=n_{\mathrm{p}} A P(Q) S^{\prime}(Q),
$$

where $n_{\mathrm{p}}$ is the number density of dendrimer molecules, $A$ denotes the contrast of the scattering length between solute particle and solvent, $P(Q)$ is the average form factor normalized at $Q=0$, and $S^{\prime}(Q)$ is the apparent structure factor given by $S^{\prime}(Q)=1+\beta(Q)[S(Q)-1]$, where $\beta(Q)$ is the polydispersity factor [46] that incorporates the size and shape fluctuations into the analysis and $S(Q)$ is the interparticle structure factor. The functional form of $\beta(Q)$ depends on the model of polydispersity and can be found in the Appendix for all models we use in this work. The $S(Q)$ is calculated by the Percus-Yevick closure of the Ornstein-Zernike equation [47] with a Gaussian pair potential written as [9]

$$
V(r)=V_{0} \exp \left(-\frac{3 r^{2}}{4 R_{\mathrm{g}}^{2}}\right),
$$

where $V_{0}$ characterizes the strength of the interparticle repulsion.

We first explore the size fluctuation by simply modeling the fluctuating dendrimers as a collection of polydisperse spheres. Assuming a Gaussian distribution for the sphere size, $P(Q)$ is expressed as $[45,48]$

$$
\begin{aligned}
P_{\mathrm{S}}(Q)= & \sqrt{\frac{2}{\pi}} \frac{1}{\sigma_{R}}\left[1+\operatorname{erf}\left(\frac{R}{\sqrt{2} \sigma_{R}}\right)\right]^{-1} \\
& \times \int_{0}^{\infty}\left(\frac{3 j_{1}(Q r)}{Q r}\right)^{2} \exp \left(-\frac{(r-R)^{2}}{2 \sigma_{R}^{2}}\right) d r \\
& +a_{\mathrm{b}} P_{\mathrm{b}}(Q),
\end{aligned}
$$

where $\left[3 j_{1}(Q r) / Q r\right]^{2}$ is the form factor of a sphere with radius of $r, j_{1}(x)$ is the first-order spherical Bessel function of the first kind, $P_{\mathrm{b}}(Q)$ represents the contribution from the intraparticle density variation with $a_{\mathrm{b}}$ denoting its amplitude $[45,49,50], R$ is the average radius of the polydisperse spheres, and $\sigma_{R}$ is the standard deviation of the Gaussian distribution of 

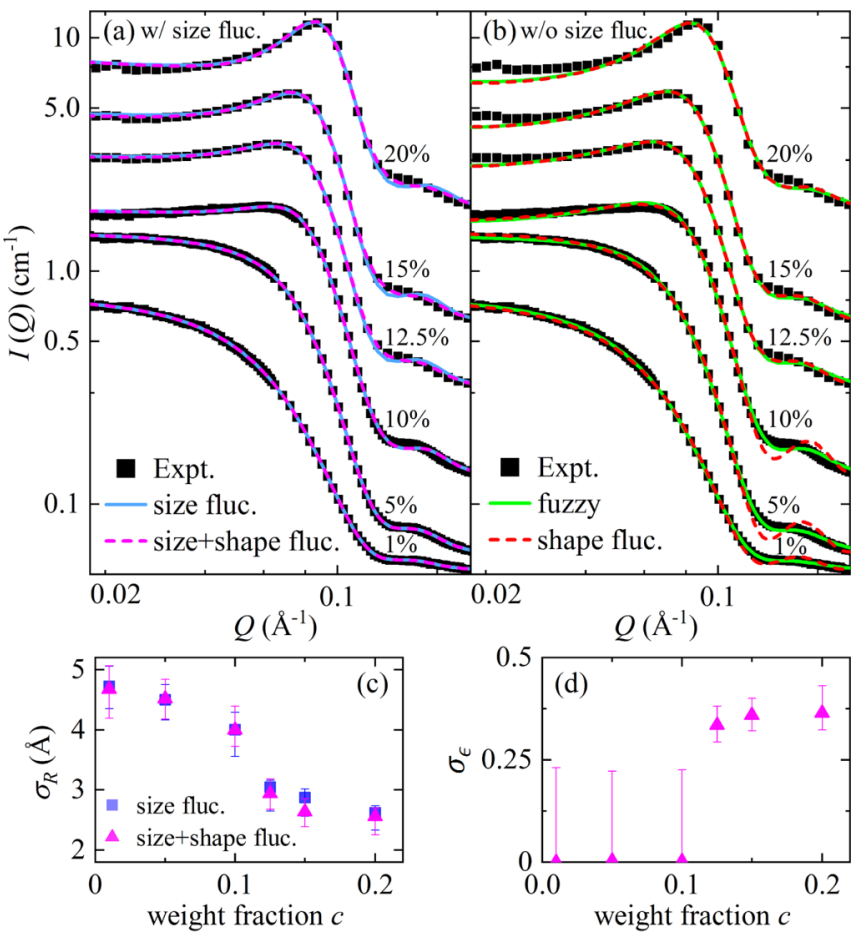

FIG. 2. SANS analyses of the G6 PAMAM dendrimers dissolved in $\mathrm{D}_{2} \mathrm{O}$ at $c=1,5,10,12.5,15$, and 20 wt.\%. Symbols in (a) and (b) denote the measured spectra (they are vertically shifted for better visibility). (a) Fits with the size fluctuation model and the model with both the size and shape fluctuations. (b) Fits with the monodisperse fuzzy-ball model and the model only considering the shape fluctuation. These two models, without explicitly considering the size fluctuation, underestimate the low- $Q$ intensity at $c \geqslant 10 \mathrm{wt} . \%$. (c) Size fluctuation $\sigma_{R}$ of the G6 dendrimer as a function of $c$. The results are obtained with the size fluctuation model and the model with both the size and shape fluctuations. (d) Shape fluctuation $\sigma_{\epsilon}$ of the G6 dendrimer as a function of $c$. More details of the SANS fitting can be found in the Supplemental Material [54].

$r ; \sigma_{R}$ reflects the fluctuating amplitude of the dendrimer size. This form factor ignores the fuzzy profile of the radial density distribution $[4,45]$. It could be reasonable for high-generation dendrimers, because their ratios of the fuzzy edge $\sigma_{\mathrm{f}}$ [see Eq. (4)] to radius are relatively small [45]. The analyses for the G6 samples are displayed in Fig. 2(a) (blue solid lines). The fitting quality is very satisfactory at the measured concentrations, especially considering the simplicity of the model.

To highlight the effect of the size fluctuation, we analyze the same data with the frequently used monodisperse fuzzyball model [39,45,51-53]. Here the size fluctuation is not considered, so $\beta(Q)=1$ and $S^{\prime}(Q)=S(Q)$. In addition, $P(Q)$ is given by [45]

$$
P_{\mathrm{f}}(Q)=\left[\frac{3 j_{1}(Q R)}{Q R} \exp \left(-\frac{Q^{2} \sigma_{\mathrm{f}}^{2}}{4}\right)\right]^{2}+a_{\mathrm{b}} P_{\mathrm{b}}(Q),
$$

where $\sigma_{\mathrm{f}}$ denotes the spatial range of the fuzzy edge. This model has the same number of parameters as the preceding one. The fitting results, shown in Fig. 2(b) (green solid lines), exhibit clear discrepancies from the measured spectra at $c \geqslant 10 \mathrm{wt} \%$. The low- $Q$ intensity is remarkably underesti- mated. The peak position also deviates from the measured one. Comparing the two models, we assert that the size fluctuation is significant and should be taken into account in analyzing the SAS data at $c \geqslant 10 \mathrm{wt} \%$. Numerically, the incorporation of the size fluctuation lowers the low- $Q$ part of $\beta(Q)$ from 1 since $\beta(Q=0)=\left\langle r^{3}\right\rangle^{2} /\left\langle r^{6}\right\rangle$ [46] and thus lifts the low- $Q$ part of $S^{\prime}(Q)$ to match the experimental data. Notice that, at $c \leqslant 5 \mathrm{wt} . \%$, both models work well, leading to contradictory results on the existence of the size fluctuation at this concentration regime. This issue will be clarified in the scattering contrast analysis shown later.

Besides the size fluctuation, the shape fluctuation has also been reported for ultrasoft colloidal particles [18,55,56]. In particular, simulation results suggest that the dendrimer molecule can deform to a spheroidlike form [57]. Therefore, we refine the polydisperse sphere model by incorporating the shape fluctuation effect. The overall shape of a dendrimer molecule is modeled as a spheroid with semiaxes $a$, $a$, and $\epsilon a$, where $\epsilon$ is the aspect ratio. In this model, the particle volume obeys a Gaussian distribution and $\epsilon$ obeys a Schultz distribution [46] with the center at 1 and the standard deviation of $\sigma_{\epsilon}$ (see the Appendix for the distribution functions). The fitting results of this model are given in Fig. 2(a) (magenta dashed lines). It can be seen that additionally incorporating the shape fluctuation improves the fit at high $Q$. Notice that solely incorporating the shape fluctuation but without the size fluctuation cannot match the low- $Q$ spectra [Fig. 2(b) (red dashed lines)], since in this case $\beta(Q \rightarrow 0)$ does not deviate from 1 .

Figures 2(c) and 2(d) show the fitting results for $\sigma_{R}$ and $\sigma_{\epsilon}$ of the G6 dendrimer, respectively. At the dilute limit, $\sigma_{R}$ is $4.7 \AA$, corresponding to a dynamic polydispersity $\xi_{R}=\sigma_{R} / R$ of $15 \%$. As $c$ crosses about $11 \mathrm{wt} \%$, the size fluctuation is suppressed while the shape fluctuation is strongly enhanced. Later we will show that this behavior corresponds to a dynamic crossover.

Seeing that the fuzzy-ball model has been widely adopted in previous SAS studies of soft particles [39,53], one may be interested in an analysis combining both the size polydispersity and the fuzziness. We perform this calculation and find that it again confirms the evolution of the size fluctuation as displayed in Fig. 2(c). Additionally, it finds the $\sigma_{\mathrm{f}}$-to- $R$ ratio of the G6 dendrimer to be about $27 \%$. This value is much smaller than that of the lower-generation samples shown later. As for the fitting quality, this model performs a little worse than the preceding one which incorporates both the size and shape fluctuations. Therefore, we conclude that the G6 dendrimer is featured better by a clear boundary than by a fuzzy periphery. Details of this analysis are given in the Supplemental Material [54].

All the above models highlight the important role of the size fluctuation in decoding the structure of ultrasoft colloids. Even so, it is crucial to verify its existence without explicitly involving any SANS model. Since the dendrimer has a water-accessible architecture [58,59], the contrast term $A$ in Eq. (1) is written as $A=\left\langle\left(b_{\mathrm{pol}}+N b_{\mathrm{w}}-n_{\mathrm{w}} V_{\mathrm{p}} b_{\mathrm{w}}\right)^{2}\right\rangle$, where $b_{\text {pol }}$ is the total scattering length of a dry dendrimer, $b_{\mathrm{w}}$ is the average scattering length of a water molecule, $N$ is the number of water molecules inside a dendrimer, $V_{\mathrm{p}}$ is the volume of a dendrimer in solution, $n_{\mathrm{w}}$ is the number density of bulk water, and $\langle\cdots\rangle$ denotes the average over all particles. It is 

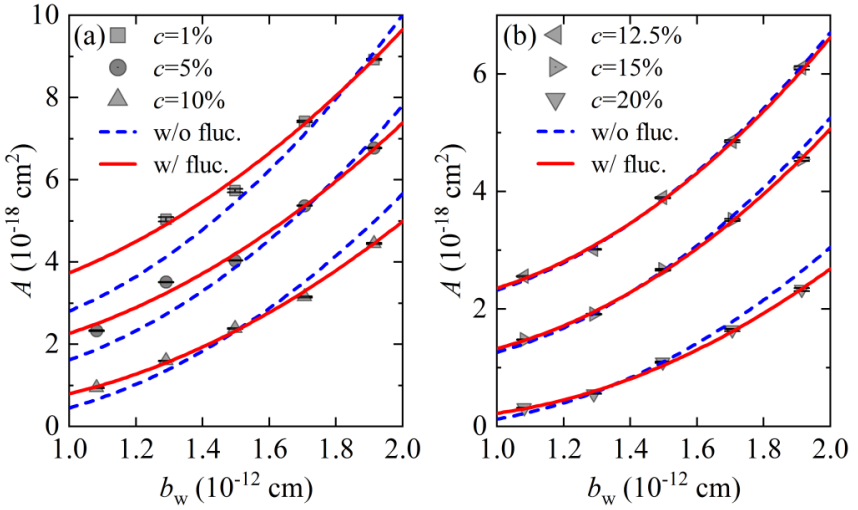

FIG. 3. Scattering contrast $A$ as a function of the average scattering length of solvent molecule $b_{\mathrm{w}}$ at (a) $c \leqslant 10 \mathrm{wt} . \%$ and (b) $c>10$ wt. $\%$. Symbols denote the experimental results. Solid and dashed lines denote the fits using Eq. (6) (with size fluctuation) and Eq. (7) (without size fluctuation), respectively. The data are shifted vertically for better visibility. More details of the calculation can be found in the Supplemental Material [54].

straightforward to find that

$$
\begin{aligned}
A\left(b_{\mathrm{w}}\right)= & {\left[n_{\mathrm{w}}^{2}\left(\left\langle V_{\mathrm{p}}\right\rangle^{2}+\left\langle\Delta V_{\mathrm{p}}^{2}\right\rangle\right)+\langle N\rangle^{2}+\left\langle\Delta N^{2}\right\rangle\right.} \\
& \left.-2 n_{\mathrm{w}}\left(\left\langle V_{\mathrm{p}}\right\rangle\langle N\rangle+\left\langle\Delta V_{\mathrm{p}} \Delta N\right\rangle\right)\right] b_{\mathrm{w}}^{2} \\
& -2 b_{\mathrm{pol}}\left(n_{\mathrm{w}}\left\langle V_{\mathrm{p}}\right\rangle-\langle N\rangle\right) b_{\mathrm{w}}+b_{\mathrm{pol}}^{2},
\end{aligned}
$$

where $\Delta V_{\mathrm{p}}=V_{\mathrm{p}}-\left\langle V_{\mathrm{p}}\right\rangle$ and $\Delta N=N-\langle N\rangle$. The $\Delta N$ and $\Delta V_{\mathrm{p}}$ should be highly correlated. To the first-order approximation, it can be assumed that $\Delta N$ is proportional to $\Delta V_{\mathrm{p}}$ by $\Delta N=n_{\text {in }} \Delta V_{\mathrm{p}}[60]$, ${ }^{1}$ where $n_{\text {in }}$ should be smaller than $n_{\mathrm{w}}$ due to the excluded volume of the constituent atoms of the dendrimer. Thus Eq. (5) is rewritten as

$$
A\left(b_{\mathrm{w}}\right)=\left(\alpha^{2}+\theta^{2}\right) b_{\mathrm{w}}^{2}-2 b_{\mathrm{pol}} \theta b_{\mathrm{w}}+b_{\mathrm{pol}}^{2},
$$

where $\theta=n_{\mathrm{w}}\left\langle V_{\mathrm{p}}\right\rangle-\langle N\rangle$ and $\alpha=\left(n_{\mathrm{w}} / n_{\text {in }}-1\right) \sigma_{N}$, with $\sigma_{N}=$ $\sqrt{\left\langle\Delta N^{2}\right\rangle}$ denoting the fluctuation of the number of invasive water molecules. If no such fluctuation exists, then $\alpha=0$ and Eq. (6) reduces to

$$
A\left(b_{\mathrm{w}}\right)=\left(\theta b_{\mathrm{w}}-b_{\mathrm{pol}}\right)^{2} .
$$

Equations (6) and (7) provide an approach to verify the existence of the size fluctuation (proportional to $\sigma_{N}$ ) from a microscopic view. One can vary $b_{\mathrm{w}}$ by changing the molar fraction of $\mathrm{D}_{2} \mathrm{O}$ in solvent $(\gamma)$ and fit the experimental $A\left(b_{\mathrm{w}}\right)$ with Eqs. (6) and (7). If the size fluctuation is considerable, the fitting quality with Eq. (6) will be better than that with Eq. (7). We vary $\gamma$ from $100 \%$ to $60 \%$ for all concentrations and fit the experimental contrast term with Eqs. (6) and (7). The results are shown in Fig. 3. It can be seen that at $c \leqslant 10 \mathrm{wt} . \%$, Eq. (6) performs much better than Eq. (7), suggesting that the fluctuations of size and invasive water exist and strongly affect the scattering contrast. At $c>10 \mathrm{wt} . \%$, this effect is

\footnotetext{
${ }^{1}$ Note that the density of nanoconfined water does not change significantly with pressure. This is confirmed experimentally. Considerable changes only happen at temperatures lower than $220 \mathrm{~K}$ and pressures higher than about 1 kbar. See Ref. [60].
}

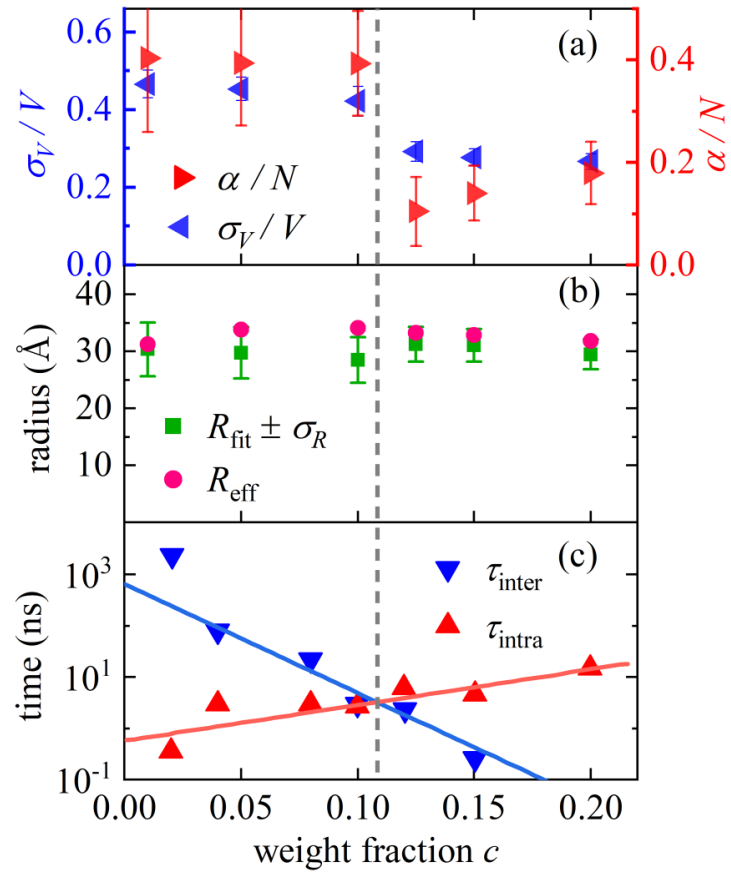

FIG. 4. (a) Fluctuations of volume $\left(\sigma_{V} / V_{\mathrm{p}}\right)$ and invasive water $\left(\alpha / N \propto \sigma_{N} / N\right)$ of a G6 dendrimer as a function of $c$. (b) Radius of the G6 dendrimer as a function of $c$. Here $R_{\text {fit }}$ is obtained by fitting with the size fluctuation model and $R_{\text {eff }}$ is the effective radius defined by the condition of compressibility equivalence between the soft particle and the hard sphere [61]. (c) Interparticle collisional time $\tau_{\text {inter }}$ and intraparticle relaxation time $\tau_{\text {intra }}$ of G6 dendrimers as a function of $c$ [62]. The dashed line marks the crossover concentration $c^{*}$.

less significant, implying a smaller fluctuation. The values of $\alpha / N$, representing the fluctuation of invasive water, are given in Fig. 4(a). In the same panel, we also plot the particle volume fluctuation $\sigma_{V} / V_{\mathrm{p}}$ obtained from the model fitting of SANS spectra as illustrated in Fig. 2(a). It is remarkable that these two quantities display very similar behaviors, especially considering that they are found with different approaches. Such consistency confirms the existence of the size fluctuation in the G6 dendrimer.

As mentioned above, SAS analyses on neutral ultrasoft colloids usually deteriorate when $c$ is larger than about $10 \mathrm{wt} \%$. It has been tentatively attributed to the failure of factorizing $I(Q)$ into the product of $P(Q)$ and $S(Q)$ due to the interparticle interpenetration or overlap $[4,7,63]$. Nevertheless, this concentration is much lower than the one at the physical overlap, $c_{\mathrm{O}} \approx 47$ wt. $\%$, defined by $c_{\mathrm{O}} \approx m_{0} \phi_{\text {rcp }} / \frac{4}{3} \pi R^{3} \rho$, where $m_{0}$ is the mass of a dendrimer molecule, $\phi_{\mathrm{rcp}}=0.637$ is the random-close-packing volume fraction [64], $\rho$ is the solution density, and $R$ is the radius of an isolated dendrimer. Many studies show that strong interpenetration or overlap does not occur at such low concentrations [65-71]. Our analysis suggests that the failure of $P(Q) \cdot S(Q)$ factorization is due to the molecular fluctuation and can be corrected with Eq. (1). In fact, Pedersen also found that the SANS intensity of block copolymer micelles is expressed by Eq. (1) rather than a product of $P(Q)$ and $S(Q)$ by considering the configuration distribution of the chains in the corona [51]. We argue that the particle conformational fluctuation is the basis for both 
Ref. [51] and our work. This agreement suggests that Eq. (1) is the better expression to account for the softness, which originates from the flexible architecture, of ultrasoft particles such as dendrimers and starlike polymers. It should be pointed out that Eq. (1) is based on the assumption that the particle conformation decouples with the position [46]. It might become invalid at concentrations close to random close packing because of the spatial heterogeneity of particle deformation predicted in a recent simulation study [19].

As seen from Figs. 2 and 4(a), all discussed fluctuations exhibit crossovers at $c^{*} \approx 11 \mathrm{wt} . \%$. We first explore the role of the osmotic pressure in this crossover. The osmotic pressure could affect the molecular conformation of ultrasoft colloids $[61,72,73]$ and such an effect may lead to a change in fluctuations. Recently, it was found that even at concentrations well below $\phi_{\text {rcp }}$, the osmotic pressure can induce the shrink of the ultrasoft particle, which is reflected by the decrease of the effective particle radius $R_{\text {eff }}$ defined by the condition of an osmotic compressibility equivalence between soft particles and hard spheres [61]. We calculate the $R_{\text {eff }}$ of our sample and show the result in Fig. 4(b). In addition, we plot the values of $R$ fitted with the size fluctuation model in the same panel. Neither radii exhibits any significant change around $c^{*}$, indicating that the observed crossovers should not be attributed to the conformational variation induced by the osmotic effect. We thus turn to seek the dynamic origin of this phenomenon. Figure 4(c) shows the results of a previous NSE study on the same system [62]. By decomposing the motion of a dendrimer into translational diffusion and internal relaxation, this NSE analysis gives two characteristic times: the interparticle collisional time $\tau_{\text {inter }}$ and the intraparticle relaxation time $\tau_{\text {intra }}$. Interestingly, $\tau_{\text {inter }}$ and $\tau_{\text {intra }}$ also intersect at $c^{*}$. So we associate the fluctuation crossover with the dynamic process. At $c<c^{*}$, conformational fluctuations emerge due to the enormous internal degrees of freedom. The interparticle collisions do not strongly perturb the internal relaxation since $\tau_{\text {intra }}<\tau_{\text {inter }}$. In contrast, at $c>c^{*}$ and $\tau_{\text {intra }}>\tau_{\text {inter }}$, the frequent collisions hinder the dendrimer from fully exploring its conformational space at the dilute limit and thus restrain the size fluctuation. Meanwhile, these collisions enhance the particle deformation and suppress the internal relaxation, which results in an increasing shape fluctuation.

We further measure the G4 samples to investigate the structural origin of the observed fluctuations. The polydisperse sphere model and monodisperse fuzzy-ball model are applied to fit the SANS spectra and the results are shown in Fig. 5(a). In contrast to the G6 samples, for G4 samples the fuzzy-ball model works well, while the polydisperse sphere model overestimates the low- $Q$ intensity and gives an inappropriate oscillation at high $Q$. This result indicates that the size fluctuation is imperceptible for G4 samples. To confirm this observation, we perform the contrast analysis given by Eqs. (5)-(7). As seen from Fig. 5(b), Eqs. (6) and (7) give the same fits, showing that the size fluctuation term $\alpha$ equals zero. The sharp difference between the G4 and G6 samples reveals the role of molecular structure in determining the fluctuations. For our G4 sample, the ratio of the fuzzy edge to the radius $\left(\sigma_{\mathrm{f}} / R\right)$ is $74 \%$, which demonstrates the pronounced open feature of its periphery. Intuitively, the strong fuzziness implies an unsharp boundary, which makes the particle volume and its
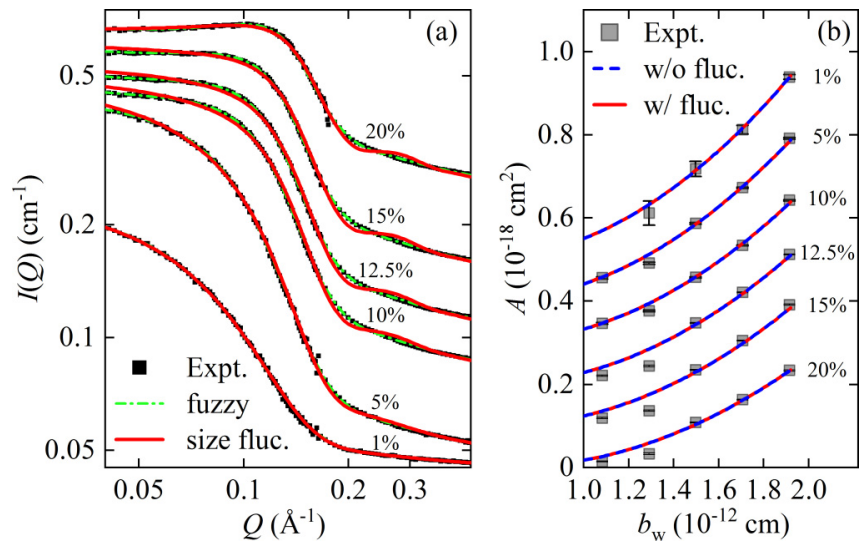

FIG. 5. (a) SANS analysis of G4 PAMAM dendrimers dissolved in $\mathrm{D}_{2} \mathrm{O}$ at $c=1,5,10,12.5,15$, and $20 \mathrm{wt} \%$. Symbols denote the measured spectra. Solid and dashed lines denote the fits using the monodisperse fuzzy-ball model and the size fluctuation model, respectively. (b) Scattering contrast of G4 dendrimers as a function of $b_{\mathrm{w}}$. Symbols denote the experimental results. Solid and dashed lines denote the fits using Eqs. (6) and (7), respectively. Data are vertically shifted for better visibility.

fluctuation not so well defined. In contrast, G6 dendrimers are found to be alike spheres with a clearer boundary. This result agrees with the previous finding that the dendrimer structure evolves from an open one to a compact one as the generation increases $[45,57,59,74,75]$. The evident compactness in G6 dendrimers leads to small intraparticle free volume, which enhances the steric interaction between dendrons $[4,70,76]$. Therefore, it is reasonable that the dendrons can fluctuate collectively with long-range correlation and form the size fluctuation of the whole particle. For G4 dendrimers, a simulation shows that the internal fluctuations are uncorrelated at large distances [24] due to the open structure. In this case, intraparticle motions involving long-range correlated motion of monomers, such as the size fluctuation, should be weak. With these observations and discussion, we suggest that the backfolding of dendrimer branches is important in forming the size fluctuation. The monomers in the outer shells of a dendrimer backfold and fill the internal voids. As shown in many studies, these backfolded monomers are distributed throughout the whole molecule $[4,59,75,77]$. Thus, the backfolding and associated unfolding motions are possible to induce considerable conformational changes. Previous simulations have proven that higher-generation dendrimers undergo a larger extent of backfolding due to their compact outer shells $[59,75]$. The collective motion of these backfolded monomers can give rise to a larger size fluctuation for higher-generation samples. This is in agreement with our findings with the G4 and G6 dendrimers.

\section{CONCLUSION}

In summary, we have investigated the fluctuation of ultrasoft colloids by using PAMAM dendrimers as the model system. By considering the dynamic polydispersity and the fluctuation of invasive water in SANS analysis, we revealed the fluctuating amplitudes of the size and shape of the G6 dendrimer. The size fluctuation was found to be of particular 
importance in interpreting the SAS data. These fluctuations exhibit strong dependences on the dynamics and structure. The competition between the intra- and interparticle dynamics introduces a crossover concentration above which the size fluctuation is suppressed and the shape fluctuation increases. The absence of size fluctuation in G4 dendrimers highlights the importance of the structural compactness in determining the intraparticle motion. Our approach should not be limited to dendrimers, but is also applicable to a wide range of soft particle systems and provides a basis for further exploration of the fluctuation effect on various structural and dynamic properties.

\section{ACKNOWLEDGMENTS}

This research was supported by the National Natural Science Foundation of China (Grants No. 11975136 and No. U1830205). We are grateful to the EQ-SANS at Spallation Neutron Source, Oak Ridge National Laboratory and the D22 beamline at Institut Laue-Langevin for beamtime.

\section{APPENDIX: SANS MODELS}

To quantitatively interpret the SANS spectra with Eq. (1), it is necessary to obtain the expressions of the form factor $P(Q)$, the polydispersity factor $\beta(Q)$, and the structure factor $S(Q)$. The $S(Q)$ is numerically calculated by solving the Ornstein-Zernike equation within the Percus-Yevick closure involving the Gaussian effective pair potential [Eq. (2)]. In this Appendix we give the details of $P(Q)$ and $\beta(Q)$ of various polydisperse models used in this work.

The form factor of the dendrimer can be expressed in the form

$$
P(Q)=P_{0}(Q)+a_{\mathrm{b}} P_{\mathrm{b}}(Q),
$$

where $P_{\mathrm{b}}(Q)$ dominates the high- $Q$ regime and is written as [45]

$$
P_{\mathrm{b}}(Q)=\frac{\sin \left[\mu \tan ^{-1}\left(q_{\mathrm{b}}^{*}\right)\right]}{\mu q_{\mathrm{b}}^{*}\left(1+q_{\mathrm{b}}^{* 2}\right)^{\mu / 2}},
$$

where $q_{\mathrm{b}}^{*}=Q \xi /\left[\operatorname{erf}\left(\frac{Q R_{\mathrm{g}}}{\sqrt{6}}\right)\right]^{3}, \mu=v^{-1}-1, v$ is the FloryHuggins parameter, and $\xi$ is the correlation length of the intradendrimer density fluctuations. As suggested by Rathgeber et al. [45], in this work $v$ is set equal to 0.6 and $\xi$ is set to $15 \AA$.

Here $P_{0}(Q)$ represents the scattering contribution from the overall shape of dendrimers, which has different expressions for different models. The general form of $P_{0}(Q)$ is written as

$$
P_{0}(Q)=\left\langle|F(\boldsymbol{Q})|^{2}\right\rangle /\left\langle|F(\boldsymbol{Q}=\mathbf{0})|^{2}\right\rangle,
$$

where $F(\boldsymbol{Q})$ is the total scattering amplitude of a single particle defined as

$$
F(\boldsymbol{Q})=\int_{V_{\mathrm{p}}} \exp (i \boldsymbol{Q} \cdot \boldsymbol{x}) \Delta \rho(\boldsymbol{x}) d \boldsymbol{x} .
$$

The above integral is over the particle volume $V_{\mathrm{p}}$ and $\Delta \rho(\boldsymbol{x})$ is the contrast of the scattering length densities of solute particle and solvent at position $\boldsymbol{x}$. Here a solute particle is a complex of a dendrimer particle and its invasive water molecules.

The polydispersity factor $\beta(Q)$ is calculated from $F(\boldsymbol{Q})$,

$$
\beta(Q)=|\langle F(\boldsymbol{Q})\rangle|^{2} /\left\langle|F(\boldsymbol{Q})|^{2}\right\rangle,
$$

where $\langle\cdots\rangle$ represents the average over the distribution of particle conformations and orientations. Therefore, specifying $F(\boldsymbol{Q})$ and its distribution is the basis to calculating Eq. (1) for different SANS models. One may notice that Eq. (A5) does not involve the effect of the intradendrimer density fluctuations. To explain this, it would be helpful to revisit the apparent structure factor $S^{\prime}(Q)$ that can be rewritten as $S^{\prime}(Q)=S(Q)+[1-\beta(Q)][1-S(Q)]$. Because $S(Q)$ approaches 1 at high $Q$, the effect of $\beta(Q)$ on $S^{\prime}(Q)$ mainly appears at low $Q$. Therefore, it is reasonable to ignore the intradendrimer density fluctuations and consider only the overall shape of dendrimers in Eq. (A5).

In the following we present the expressions of $F(\boldsymbol{Q})$ for different models. With these expressions known, we can employ Eq. (1) to fit the experimental data. For the polydisperse sphere model [Eq. (3)], $F(\boldsymbol{Q})$ and its Gaussian distribution function are

$$
\begin{aligned}
& F_{\mathrm{s}}(\boldsymbol{Q})=\frac{3 j_{1}(Q r)}{Q r} \frac{4 \pi}{3} r^{3}, \\
& w_{1}(r)=c_{1} \exp \left(-\frac{(r-R)^{2}}{2 \sigma_{R}^{2}}\right),
\end{aligned}
$$

where $c_{1}$ is the normalized constant.

For the model with both the size and shape fluctuations, the overall shape of the particle is modeled as a spheroid with semiaxes $a, a$, and $b$. It is assumed that the particle volume $V_{\mathrm{p}}$ obeys a Gaussian distribution of $w_{2}\left(V_{\mathrm{p}}\right)$ and the aspect ratio $\epsilon(\epsilon=b / a)$ obeys a Schultz distribution of $w_{3}(\epsilon)$. The expressions are written as

$$
\begin{aligned}
F_{\mathrm{e}}(\boldsymbol{Q}) & =\frac{3 j_{1}\left(Q a \sqrt{\sin ^{2} \eta+\epsilon^{2} \cos ^{2} \eta}\right)}{Q a \sqrt{\sin ^{2} \eta+\epsilon^{2} \cos ^{2} \eta}} V_{\mathrm{p}}, \\
w_{2}\left(V_{\mathrm{p}}\right) & =c_{2} \exp \left(-\frac{\left(V_{\mathrm{p}}-\bar{V}_{\mathrm{p}}\right)^{2}}{2 \sigma_{V}^{2}}\right), \\
w_{3}(\epsilon) & =\frac{[(Z+1) / \bar{\epsilon}]^{Z+1} \epsilon^{Z}}{\Gamma(Z+1)} \exp \left[-\left(\frac{Z+1}{\bar{\epsilon}}\right) \epsilon\right],
\end{aligned}
$$

where $\eta$ is the angle between $\boldsymbol{Q}$ and the $b$ axis, $V_{\mathrm{p}}$ is the volume of the ellipsoid, $\bar{V}_{\mathrm{p}}$ and $\bar{\epsilon}$ are the averages of $V_{\mathrm{p}}$ and $\epsilon$, respectively, $c_{2}$ is the normalized constant, $Z=(\bar{\epsilon} / \epsilon)^{2}-1$, and $\Gamma(x)$ is the Gamma function. In this work, $\bar{\epsilon}$ is set equal to 1 based on the assumption that the average shape of the dendrimer is a sphere.

For the model only considering the shape fluctuation, we can just set $V_{\mathrm{p}}$ in Eq. (A8) as a constant in the calculation of $P_{0}(Q)$ and $\beta(Q)$. For polydisperse fuzzy-ball model, we have

$$
F_{\mathrm{f}}(Q)=\frac{3 j_{1}(Q r)}{Q r} \exp \left(-\frac{Q^{2} \sigma_{\mathrm{f}}^{2}}{4}\right) .
$$

Here $r$ is allowed to fluctuate according to the Gaussian distribution function of $w_{1}(r)$ [see Eq. (A7)].

More details regarding the SANS models and the fitting results can be found in the Supplemental Material [54]. 
[1] C. N. Likos, Soft matter with soft particles, Soft Matter 2, 478 (2006).

[2] G. S. Grest, L. J. Fetters, J. S. Huang, and D. Richter, Star polymers: Experiment, theory, and simulation, Adv. Chem. Phys. 94, 67 (1996).

[3] W. Burchard, in Branched Polymers II, edited by J. Roovers, Advances in Polymer Science (Springer, Berlin, 1999), Vol. 143, pp. 113-194.

[4] M. Ballauff and C. N. Likos, Dendrimers in solution: Insight from theory and simulation, Angew. Chem. Int. Ed. 43, 2998 (2004).

[5] A. P. Gast, Structure, interactions, and dynamics in tethered chain systems, Langmuir 12, 4060 (1996).

[6] R. G. Winkler, D. A. Fedosov, and G. Gompper, Dynamical and rheological properties of soft colloid suspensions, Curr. Opin. Colloid Interface Sci. 19, 594 (2014).

[7] C. N. Likos, H. Löwen, M. Watzlawek, B. Abbas, O. Jucknischke, J. Allgaier, and D. Richter, Star Polymers Viewed as Ultrasoft Colloidal Particles, Phys. Rev. Lett. 80, 4450 (1998).

[8] C. N. Likos, M. Schmidt, H. Löwen, M. Ballauff, D. Pötschke, and P. Lindner, Soft interaction between dissolved flexible dendrimers: Theory and experiment, Macromolecules 34, 2914 (2001).

[9] C. N. Likos, S. Rosenfeldt, N. Dingenouts, M. Ballauff, P. Lindner, N. Werner, and F. Vögtle, Gaussian effective interaction between flexible dendrimers of fourth generation: A theoretical and experimental study, J. Chem. Phys. 117, 1869 (2002).

[10] D. Vlassopoulos and M. Cloitre, Tunable rheology of dense soft deformable colloids, Curr. Opin. Colloid Interface Sci. 19, 561 (2014).

[11] H. Princen, Rheology of foams and highly concentrated emulsions: I. Elastic properties and yield stress of a cylindrical model system, J. Colloid Interface Sci. 91, 160 (1983).

[12] T. G. Mason, J. Bibette, and D. A. Weitz, Elasticity of Compressed Emulsions, Phys. Rev. Lett. 75, 2051 (1995).

[13] A. Fernandez-Nieves, H. Wyss, J. Mattsson, and D. A. Weitz, Microgel Suspensions: Fundamentals and Applications (Wiley$\mathrm{VCH}$, Weinheim, 2011).

[14] R. T. Bonnecaze and M. Cloitre, in High Solid Dispersions, edited by M. Cloitre, Advances in Polymer Science (Springer, Berlin, 2010), Vol. 236, pp. 117-161.

[15] M. Pelaez-Fernandez, A. Souslov, L. A. Lyon, P. M. Goldbart, and A. Fernandez-Nieves, Impact of Single-Particle Compressibility on the Fluid-Solid Phase Transition for Ionic Microgel Suspensions, Phys. Rev. Lett. 114, 098303 (2015).

[16] D. Richter, B. Stühn, B. Ewen, and D. Nerger, Collective Relaxation of Star Polymers-A Neutron Spin-Echo Study, Phys. Rev. Lett. 58, 2462 (1987).

[17] T. Narayanan, H. Wacklin, O. Konovalov, and R. Lund, Recent applications of synchrotron radiation and neutrons in the study of soft matter, Cryst. Rev. 23, 160 (2017).

[18] P. K. Maiti and B. Bagchi, Diffusion of flexible, charged, nanoscopic molecules in solution: Size and $p \mathrm{H}$ dependence for PAMAM dendrimer, J. Chem. Phys. 131, 214901 (2009).

[19] N. Gnan and E. Zaccarelli, The microscopic role of deformation in the dynamics of soft colloids, Nat. Phys. 15, 683 (2019).
[20] M. E. Helgeson, N. J. Wagner, and D. Vlassopoulos, Viscoelasticity and shear melting of colloidal star polymer glasses, J. Rheol. 51, 297 (2007).

[21] B. M. Erwin, M. Cloitre, M. Gauthier, and D. Vlassopoulos, Dynamics and rheology of colloidal star polymers, Soft Matter 6, 2825 (2010).

[22] T. A. Witten, P. A. Pincus, and M. E. Cates, Macrocrystal ordering in star polymer solutions, Europhys. Lett. 2, 137 (1986).

[23] E. Stiakakis, A. Wilk, J. Kohlbrecher, D. Vlassopoulos, and G. Petekidis, Slow dynamics, aging, and crystallization of multiarm star glasses, Phys. Rev. E 81, 020402 (2010).

[24] H. Harreis, C. N. Likos, and M. Ballauff, Can dendrimers be viewed as compact colloids? A simulation study of the fluctuations in a dendrimer of fourth generation, J. Chem. Phys. 118, 1979 (2003).

[25] W.-J. Li, W. Wang, X.-Q. Wang, M. Li, Y. Ke, R. Yao, J. Wen, G.-Q. Yin, B. Jiang, X. Li, P. Yin, and H.-B. Yang, Daisy chain dendrimers: Integrated mechanically interlocked molecules with stimuli-induced dimension modulation feature, J. Am. Chem. Soc. 142, 8473 (2020).

[26] Y. Zeng, P. Li, X. Liu, T. Yu, J. Chen, G. Yang, and Y. $\mathrm{Li}$, A "breathing" dendritic molecule-Conformational fluctuation induced by external stimuli, Polym. Chem. 5, 5978 (2014).

[27] M. Liu and J. M. Fréchet, Designing dendrimers for drug delivery, Pharm. Sci. Technol. Today 2, 393 (1999).

[28] P. Kesharwani, K. Jain, and N. K. Jain, Dendrimer as nanocarrier for drug delivery, Prog. Polym. Sci. 39, 268 (2014).

[29] J. F. G. A. Jansen, E. W. Meijer, and E. M. M. de Brabandervan den Berg, The dendritic box: Shape-selective liberation of encapsulated guests, J. Am. Chem. Soc. 117, 4417 (1995).

[30] K. Kitchens, M. Elsayed, and H. Ghandehari, Transepithelial and endothelial transport of poly (amidoamine) dendrimers, Adv. Drug Delivery Rev. 57, 2163 (2005).

[31] F. Badalkhani-Khamseh, A. Ebrahim-Habibi, and N. L. Hadipour, Atomistic computer simulations on multi-loaded PAMAM dendrimers: A comparison of amine- and hydroxylterminated dendrimers, J. Comput. Aided Mol. Des. 31, 1097 (2017).

[32] W.-D. Tian and Y.-Q. Ma, Theoretical and computational studies of dendrimers as delivery vectors, Chem. Soc. Rev. 42, 705 (2013).

[33] Y. Liu, V. S. Bryantsev, M. S. Diallo, and W. A. Goddard, PAMAM dendrimers undergo $\mathrm{pH}$ responsive conformational changes without swelling, J. Am. Chem. Soc. 131, 2798 (2009).

[34] B. Stark, C. Lach, B. Farago, H. Frey, C. Schlenk, and B. Stühn, Form fluctuations of carbosilane dendrimers in dilute solution: A study using neutron spin echo spectroscopy, Colloid. Polym. Sci. 281, 593 (2003).

[35] S. Rathgeber, M. Monkenbusch, J. L. Hedrick, M. Trollsås, and A. P. Gast, Starlike dendrimers in solutions: Structural properties and internal dynamics, J. Chem. Phys. 125, 204908 (2006).

[36] G. Foffi, F. Sciortino, P. Tartaglia, E. Zaccarelli, F. L. Verso, L. Reatto, K. A. Dawson, and C. N. Likos, Structural Arrest in Dense Star-Polymer Solutions, Phys. Rev. Lett. 90, 238301 (2003).

[37] G. C. Abade, B. Cichocki, M. L. Ekiel-Jeżewska, G. Nägele, and E. Wajnryb, Short-time dynamics of permeable particles in concentrated suspensions, J. Chem. Phys. 132, 014503 (2010). 
[38] X. Wang, L. Guerrand, B. Wu, X. Li, L. Boldon, W.-R. Chen, and L. Liu, Characterizations of polyamidoamine dendrimers with scattering techniques, Polymers 4, 600 (2012).

[39] M. Stieger, J. S. Pedersen, P. Lindner, and W. Richtering, Are thermoresponsive microgels model systems for concentrated colloidal suspensions? A rheology and small-angle neutron scattering study, Langmuir 20, 7283 (2004).

[40] S. Gupta, M. Camargo, J. Stellbrink, J. Allgaier, A. Radulescu, P. Lindner, E. Zaccarelli, C. N. Likos, and D. Richter, Dynamic phase diagram of soft nanocolloids, Nanoscale 7, 13924 (2015).

[41] D. A. Tomalia, A. M. Naylor, and W. A. Goddard, Starburst dendrimers: Molecular-level control of size, shape, surface chemistry, topology, and flexibility from atoms to macroscopic matter, Angew. Chem. Int. Ed. 29, 138 (1990).

[42] A. D. Meltzer, D. A. Tirrell, A. A. Jones, P. T. Inglefield, D. M. Hedstrand, and D. A. Tomalia, Chain dynamics in poly(amidoamine) dendrimers: A study of carbon13 NMR relaxation parameters, Macromolecules 25, 4541 (1992).

[43] S. R. Kline, Reduction and analysis of SANS and USANS data using IGOR Pro, J. Appl. Cryst. 39, 895 (2006).

[44] E. Abbasi, S. Aval, A. Akbarzadeh, M. Milani, H. Nasrabadi, S. Joo, Y. Hanifehpour, K. Nejati-Koshki, and R. Pashaei-Asl, Dendrimers: Synthesis, applications, and properties, Nanoscale Res. Lett. 9, 247 (2014).

[45] S. Rathgeber, M. Monkenbusch, M. Kreitschmann, V. Urban, and A. Brulet, Dynamics of star-burst dendrimers in solution in relation to their structural properties, J. Chem. Phys. 117, 4047 (2002).

[46] M. Kotlarchyk and S.-H. Chen, Analysis of small angle neutron scattering spectra from polydisperse interacting colloids, J. Chem. Phys. 79, 2461 (1983).

[47] J.-P. Hansen and I. R. McDonald, Theory of Simple Liquids: With Applications to Soft Matter (Academic Press, New York, 2013).

[48] T. J. Prosa, B. J. Bauer, and E. J. Amis, From stars to spheres: A SAXS analysis of dilute dendrimer solutions, Macromolecules 34, 4897 (2001).

[49] M. Daoud and J. Cotton, Star shaped polymers: A model for the conformation and its concentration dependence, J. Phys. (Paris) 43, 531 (1982).

[50] W. D. Dozier, J. S. Huang, and L. J. Fetters, Colloidal nature of star polymer dilute and semidilute solutions, Macromolecules 24, 2810 (1991).

[51] J. S. Pedersen, Structure factors effects in small-angle scattering from block copolymer micelles and star polymers, J. Chem. Phys. 114, 2839 (2001).

[52] W.-R. Chen, L. Porcar, Y. Liu, P. D. Butler, and L. J. Magid, Small angle neutron scattering studies of the counterion effects on the molecular conformation and structure of charged G4 PAMAM dendrimers in aqueous solutions, Macromolecules 40, 5887 (2007).

[53] S. Nöjd, P. Holmqvist, N. Boon, M. Obiols-Rabasa, P. S. Mohanty, R. Schweins, and P. Schurtenberger, Deswelling behaviour of ionic microgel particles from low to ultra-high densities, Soft Matter 14, 4150 (2018).

[54] See Supplemental Material at http://link.aps.org/supplemental/ 10.1103/PhysRevResearch.3.033271 for details of the SANS analyses and the NSE analysis.
[55] S. V. Lyulin, A. A. Darinskii, A. V. Lyulin, and M. A. J. Michels, Computer simulation of the dynamics of neutral and charged dendrimers, Macromolecules 37, 4676 (2004).

[56] S. J. Su and J. Kovac, Concentration dependence of shape fluctuations of uniform star polymers, J. Phys. Chem. 96, 3931 (1992).

[57] A. M. Naylor, W. A. Goddard, G. E. Kiefer, and D. A. Tomalia, Starburst dendrimers. 5. Molecular shape control, J. Am. Chem. Soc. 111, 2339 (1989).

[58] B. Wu, B. Kerkeni, T. Egami, C. Do, Y. Liu, Y. Wang, L. Porcar, K. Hong, S. C. Smith, E. L. Liu, G. S. Smith, and W.-R. Chen, Structured water in polyelectrolyte dendrimers: Understanding small angle neutron scattering results through atomistic simulation, J. Chem. Phys. 136, 144901 (2012).

[59] K. Karatasos, D. Adolf, and G. Davies, Statics and dynamics of model dendrimers as studied by molecular dynamics simulations, J. Chem. Phys. 115, 5310 (2001).

[60] Z. Wang, K. Ito, J. B. Leäo, L. Harriger, Y. Liu, and S.-H. Chen, Liquid-liquid phase transition and its phase diagram in deeplycooled heavy water confined in a nanoporous silica matrix, J. Phys. Chem. Lett. 6, 2009 (2015).

[61] Z. Wang, A. Faraone, P. Yin, L. Porcar, Y. Liu, C. Do, K. Hong, and W.-R. Chen, Dynamic equivalence between soft star polymers and hard spheres, ACS Macro Lett. 8, 1467 (2019).

[62] X. Li, L. E. Sánchez-Diáz, B. Wu, W. A. Hamilton, P. Falus, L. Porcar, Y. Liu, C. Do, A. Faraone, G. S. Smith, T. Egami, and W.-R. Chen, Dynamical threshold of diluteness of soft colloids, ACS Macro Lett. 3, 1271 (2014).

[63] I. Götze and C. N. Likos, Microscopic and coarse-grained correlation functions of concentrated dendrimer solutions, J. Phys.: Condens. Matter 17, S1777 (2005).

[64] G. D. Scott and D. M. Kilgour, The density of random close packing of spheres, J. Phys. D 2, 863 (1969).

[65] A. Topp, B. J. Bauer, T. J. Prosa, R. Scherrenberg, and E. J. Amis, Size change of dendrimers in concentrated solution, Macromolecules 32, 8923 (1999).

[66] C. L. Jackson, H. D. Chanzy, F. P. Booy, B. J. Drake, D. A. Tomalia, B. J. Bauer, and E. J. Amis, Visualization of dendrimer molecules by transmission electron microscopy (TEM): Staining methods and Cryo-TEM of vitrified solutions, Macromolecules 31, 6259 (1998).

[67] I. B. Rietveld and D. Bedeaux, Self-diffusion of poly(propylene imine) dendrimers in methanol, Macromolecules 33, 7912 (2000).

[68] S. Uppuluri, S. E. Keinath, D. A. Tomalia, and P. R. Dvornic, Rheology of dendrimers. I. Newtonian flow behavior of medium and highly concentrated solutions of polyamidoamine (PAMAM) dendrimers in ethylenediamine (EDA) solvent, Macromolecules 31, 4498 (1998).

[69] G. A. Pilkington, J. S. Pedersen, and W. H. Briscoe, Dendrimer nanofluids in the concentrated regime: From polymer melts to soft spheres, Langmuir 31, 3333 (2015).

[70] N. Zacharopoulos and I. G. Economou, Morphology and organization of poly(propylene imine) dendrimers in the melt from molecular dynamics simulation, Macromolecules 35, 1814 (2002).

[71] P. S. Mohanty, S. Nöjd, K. van Gruijthuijsen, J. J. Crassous, M. Obiols-Rabasa, R. Schweins, A. Stradner, and P. Schurtenberger, Interpenetration of polymeric microgels at ultrahigh densities, Sci. Rep. 7, 1487 (2017). 
[72] J. Mattsson, H. M. Wyss, A. Fernandez-Nieves, K. Miyazaki, Z. Hu, D. R. Reichman, and D. A. Weitz, Soft colloids make strong glasses, Nature (London) 462, 83 (2009).

[73] P. van der Scheer, T. van de Laar, J. van der Gucht, D. Vlassopoulos, and J. Sprakel, Fragility and strength in nanoparticle glasses, ACS Nano 11, 6755 (2017).

[74] P. M. Paulo, J. N. C. Lopes, and S. M. Costa, Molecular dynamics simulations of charged dendrimers: Low-to-intermediate half-generation PAMAMs, J. Phys. Chem. B 111, 10651 (2007).
[75] P. K. Maiti, T. Çağın, G. Wang, and W. A. Goddard, Structure of PAMAM dendrimers: Generations 1 through 11 , Macromolecules 37, 6236 (2004).

[76] M. L. Mansfield, Dendron segregation in model dendrimers, Polymer 35, 1827 (1994).

[77] S. Rosenfeldt, N. Dingenouts, M. Ballauff, N. Werner, F. Vögtle, and P. Lindner, Distribution of end groups within a dendritic structure: A SANS study including contrast variation, Macromolecules 35, 8098 (2002). 